\title{
ESTUDIO DE DEMANDA SOCIAL Y MERCADO OCUPACIONAL DE LA Escuela Profesional de Enfermería
}

\section{STUDY OF SOCIAL DEMAND AND MARKET FOR PROFESSIONAL OCCUPATIONAL NURSING SCHOOL}

\author{
Miguel Ángel Limo Vásquez*
}

\begin{abstract}
RESUMEN
$E_{m}^{1}$ presente trabajo tuvo como objetivo determinar y describir la demanda social y mercado ocupacional de la carrera profesional de enfermería. La investigación fue cuantitativa - descriptiva. Para su realización se recaudó información cuantitativa para cumplir con los objetivos y a su vez se encuesto a 1500 estudiantes entre $4^{\text {to }}$ y $5^{\text {to }}$ de educación secundaria de la ciudad de Chimbote, encontrando los siguientes resultados: Primero con respecto a la identificación del mercado ocupacional en el Perú existen 36 093 enfermeras, de los cuales el 98.09 \% se desempeña en el sector público y el $1.91 \%$ en el sector privado; Segundo, respecto a determinar la demanda social del profesional de enfermería se llegó a la conclusión que el profesional debe dominar temas de prevención y promoción de la salud; Tercero, la demanda social de la carrera profesional de enfermería en los egresados de educación secundaria corresponde al $6 \%$ de la totalidad de los encuestados.
\end{abstract}

Palabras claves: Demanda Social, Mercado ocupacional, Prevención y Promoción de la Salud

\begin{abstract}
This study aimed to determine and describe the social demand and market occupational career in nursing. The research was quantitative - descriptive. For its realization, quantitative information was raised to fulfill the objectives and in turn to 1,500 students between 4th and 5th secondary school in the city of Chimbote was surveyed and found the following results: First with respect to the identification of the labor market in Peru

* Universidad Católica Los Ángeles de Chimbote- Facultad de Ciencias Contables, Financieras y Administrativas - Escuela Profesional de Administración
\end{abstract}


there are 36093 nurses, of which $98.09 \%$ work in the public sector and $1.91 \%$ in the private sector; Second, with regard to determining the social demand of the nurse it was concluded that the professional must master issues of prevention and health promotion; Third, the social demand for nursing career in high school graduates corresponds to $6 \%$ of all respondents.

Keywords: Social Demand, occupational Market, Prevention and Health Promotion

\section{INTRODUCIÓN}

La educación es el principal instrumento para superar los problemas de inequidad, pobreza y exclusión, y alcanzar el desarrollo sostenido. En nuestro país, el sistema educativo atraviesa serios problemas que abarcan aspectos como la baja calidad del servicio, la poca pertinencia de los aprendizajes y la falta de propuestas pedagógicas que brinden una formación integral para el desarrollo de nuestros alumnos, entre otros. Sin embargo, aun superando estas limitaciones, los objetivos de la educación pueden ser distorsionados sino se da una gestión ética en las distintas instituciones y agentes del sistema educativo. (Guerrero, 2012).

La educación superior cumple papel muy importante en el desarrollo socio-económico mediante el cumplimiento de sus funciones esenciales de formación de profesionales, difusión cultural, creación intelectual e investigación científica y tecnológica. En el Perú lo observado es una débil articulación entre los centros de educación superior y el desarrollo nacional. Exceptuando a muy pocas instituciones de este nivel de enseñanza. Hay una escasa cultura de trabajo conjunto, lo que se refleja en diversos indicadores: exceso de oferta de egresados en varias profesiones; desvinculación entre los planes de desarrollo formativo y los planes de crecimiento empresarial; formación excesivamente académica y con poca práctica profesional. La mayoría de las carreras ofertadas y que tienen mayor demanda son las vinculadas a servicios; es más, son los jóvenes y no tanto los empleadores los que terminan influyendo más en la estructura de la oferta de formación. (Educared Perú, s.f).

Ahora, no siempre las carreras más buscadas por los postulantes son las más pedidas en el mercado laboral" Según Piscoya (s.f) Se debe hacer la aclaración de que por ejemplo enfermería que no es una carrera buscada sin embargo, el Perú aún no tiene el número de enfermeros necesarios para cubrir la demanda de necesidades en salud de la población.

Las exigencias derivadas del proceso de globalización, los nuevos perfiles demográficos y epidemiológicos en el país, las demandas y necesidades sociales por mejores condiciones de vida y salud de la población y el desarrollo científico-tecnológico constituyen un desafío para enfermería y especialmente para las instituciones responsables de la formación profesional. Procesos para planificar la oferta nacional de pre y post grado de enfermería, para desarrollar la gestión educativa, la autoevaluación y acreditación de los programas de formación, así como para direccionar la investigación de enfermería en el país, requieren ser potenciados. Por tanto, la ULADECH Católica en su mejora continua, y líder en la 
formación de talento humano para el bien común ha elaborado el estudio de la demanda social y mercado ocupacional versión III de la carrera profesional de Enfermería, el cual tiene como objetivos:

- Identificar el mercado ocupacional actual, considerando las tendencias ocupacionales a nivel nacional y regional.

- Determinar la demanda social del profesional de enfermería en los servicios de salud de la jurisdicción de la ULADECH Católica- Sede Central Chimbote.

- Determinar la demanda social de la carrera profesional de Enfermería de los egresados de educación secundaria de la ciudad de Chimbote.

\section{MATERIALES Y MÉTODOS}

La investigación fue cuantitativa y de diseño documental-descriptivo. Para el recojo de información se han utilizado la revisión documental y aplicación de encuesta, las mismas que han estado en función al objeto de estudio y los objetivos específicos de la investigación de mercado. Los resultados de la investigación se han obtenido de la aplicación de encuestas y la recolección de información, procurando que estos sean lo más exhaustivos y de la mayor actualidad posible.

\section{RESULTADOS}

a) Resultado respecto al objetivo específico $N^{\circ}$ 1: Identificar el mercado ocupacional actual, considerando las tendencias ocupacionales a nivel nacional y regional.

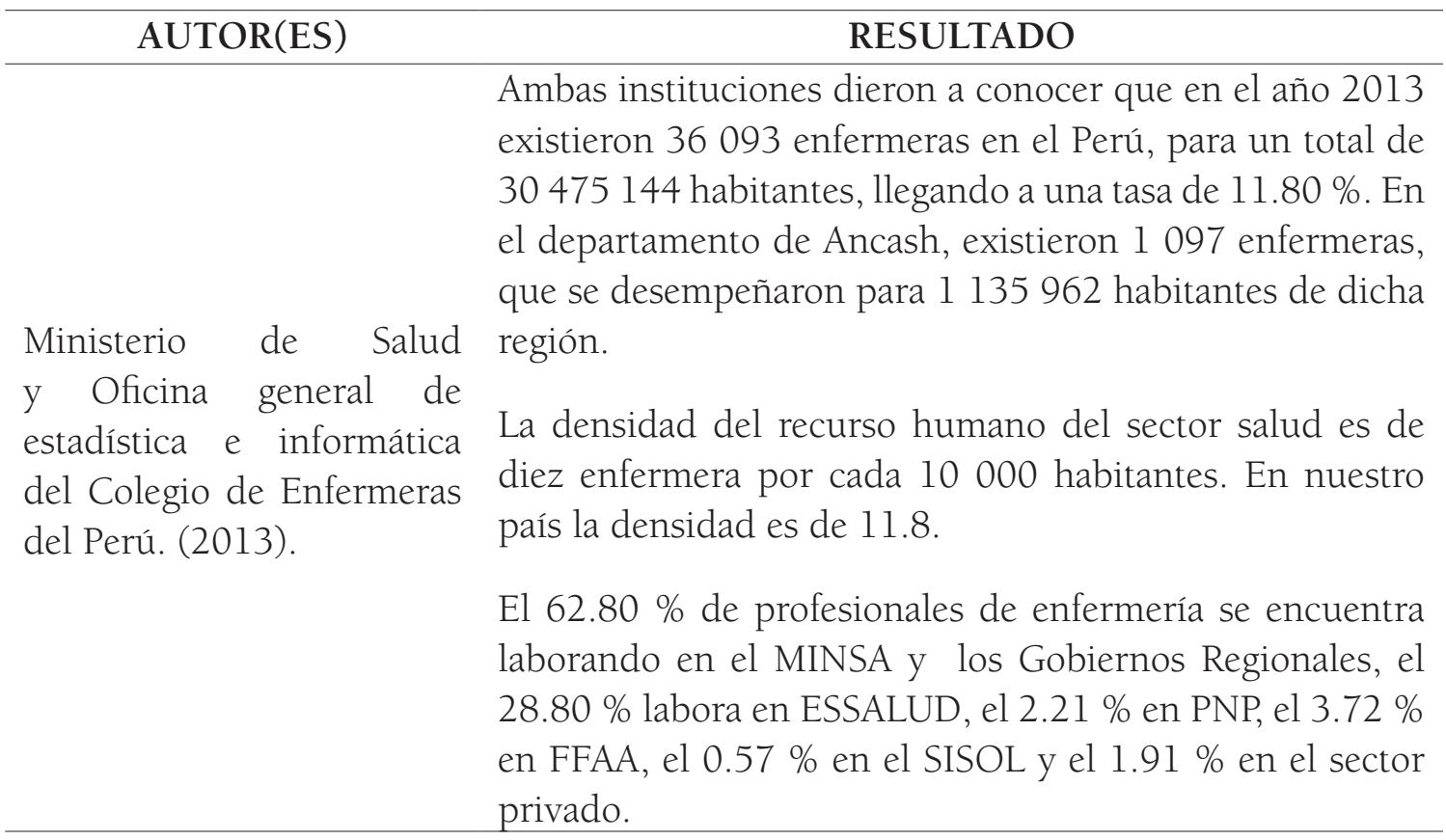


b) Resultado respecto al objetivo específico $\mathrm{N}^{\circ}$ 2: Determinar la demanda social del profesional de Enfermería en los servicios de Salud de la jurisdicción de la ULADECH Católica - Sede Central Chimbote.

\begin{tabular}{|c|c|}
\hline AUTOR(ES) & RESULTADO \\
\hline $\begin{array}{l}\text { Colegio Internacional de } \\
\text { enfermería. (2013). }\end{array}$ & $\begin{array}{l}\text { En su trabajo de investigación se habla sobre la búsqueda } \\
\text { sistemática y el aporte nuevos conocimientos para el } \\
\text { beneficio de los pacientes, familias y comunidades. } \\
\text { Abarcando temas de salud, como promoción y prevención } \\
\text { de la salud, el cuidado de las personas en todas las edades. } \\
\text { El CIE ha identificado en dos amplios sectores la prioridad } \\
\text { de la investigación de enfermería: los servicios de salud y } \\
\text { enfermedad con la prestación de los cuidados. } \\
\text { Aproximadamente el } 60 \text { \% del personal de salud de los } \\
\text { países de Latinoamérica es personal de enfermería. }\end{array}$ \\
\hline Cabrera, C. (2013) & $\begin{array}{l}\text { Manifiesta que el profesional de enfermería es muy } \\
\text { requerido en solo a nivel nacional, sino a nivel mundial. } \\
\text { Enfermería es una profesión con futuro, pues siempre } \\
\text { se necesitara profesionales especializados en salud. Un } \\
\text { profesional de enfermería tiene la opción de trabajar en } \\
\text { una entidad pública o privada, como también puede } \\
\text { formar su propia empresa. }\end{array}$ \\
\hline Guerrero, D. (2012 ) & $\begin{array}{l}\text { Manifestó que la carrera de enfermería tiene dos disciplinas: } \\
\text { El área gerontología, que estudia el proceso de } \\
\text { envejecimiento a partir del ámbito psicológico, físico y } \\
\text { sociocultural. } \\
\text { El área de la geriatría, se encarga de atender los aspectos } \\
\text { clínicos, preventivos, terapéuticos en situación de salud y } \\
\text { enfermedad. } \\
\text { Un profesional especializado en estas dos áreas podrá } \\
\text { desempeñarse en cualquier centro de salud público o } \\
\text { privado. } \\
\text { Un profesional de enfermería con conocimiento de } \\
\text { gerontología es muy reconocido en países como España, } \\
\text { Italia, EE.UU, Canadá y Suiza, ya que existe mayor } \\
\text { población de adultos mayores. }\end{array}$ \\
\hline
\end{tabular}


c) Resultado respecto al objetivo específico $N^{\circ}$ 03: Determinar la demanda social de la carrera profesional de Enfermería de los egresados de educación secundaria de la ciudad de Chimbote.

\begin{tabular}{|c|c|}
\hline ÍTEMS & RESULTADO \\
\hline $\begin{array}{l}\text { 1. ¿Después de } \\
\text { culminar tus estudios } \\
\text { secundarios, tienes } \\
\text { pensado seguir una } \\
\text { carrera profesional? }\end{array}$ & $\begin{array}{l}\text { El } 95 \text { \% de los encuestados manifestó que si tiene pensado } \\
\text { seguir un carrera profesional, mientras que el } 5 \% \text { manifestó } \\
\text { no tener interés de continuar una carrera profesional. }\end{array}$ \\
\hline $\begin{array}{l}\text { 2. ¿Qué universidad } \\
\text { elegiría para seguir su } \\
\text { carrera profesional? }\end{array}$ & $\begin{array}{l}\text { El } 38 \% \text { de los estudiantes encuestados manifestó que la } \\
\text { Universidad Nacional del Santa sería la mejor opción para } \\
\text { continuar sus estudios universitarios, mientras que un } 31 \\
\% \text { prefiere continuar sus estudios en la ULADECH Católica, } \\
\text { un } 10 \% \text { opta por continuar sus estudios universitarios en } \\
\text { la Universidad Privada San Pedro, un } 4 \% \text { en la Universidad } \\
\text { Alas Peruanas, un } 3 \% \text { en la Universidad Cesar Vallejo y } \\
\text { un } 14 \% \text { prefiere estudiar en otras universidades. }\end{array}$ \\
\hline $\begin{array}{l}\text { 3. ¿Qué carrera } \\
\text { profesional te gustaría } \\
\text { estudiar? }\end{array}$ & $\begin{array}{l}\text { En este Ítems se obtuvo como información que el } 9 \% \\
\text { de los encuestados opta por la carrera de ingeniería } \\
\text { Civil, mientras que un } 7 \% \text { se inclina para la carrera a de } \\
\text { administración de empresa, un } 6 \% \text { prefiere estudiar la } \\
\text { carrera profesional de enfermería. }\end{array}$ \\
\hline
\end{tabular}

\section{DISCUSIÓN}

\subsection{Discusión de los resultados del objetivo específico $\mathrm{N}^{\circ} 1$}

Ambas instituciones afirman que existe una sobreoferta de profesionales de enfermería a nivel nacional. A nivel departamental, Áncash tiene una densidad de 9.7, llegándose a la conclusión que existe una mala distribución de profesionales de la salud.

El mayor número de profesionales de enfermería se desempeña en el sector público, dejando solo un $1.91 \%$ de profesionales desempeñarse en el sector privado.

\subsection{Discusión de los resultados del objetivo específico $\mathrm{N}^{\circ} 2$ :}

El Colegio Internacional de Enfermería (2013) hace mención sobre la importancia de la promoción y prevención en la salud.

Guerrero (2012) al igual que el Colegio Internacional de Enfermería mencionan sobre la importancia de la gerontología en la carrera de enfermería. En países extranjeros es demandado dicho profesional con especialización en gerontología. 
Cabrera (2013), en unión con el colegio Internacional de Enfermería manifiesta que el profesional de enfermería es muy requerido a nivel mundial. La carrera de enfermería es una profesión con futuro, debido a la necesidad de profesionales en salud.

\subsection{Discusión de los resultados del objetivo específico $N^{\circ} 3$ :}

Según la encuesta aplicada a los estudiantes del $4^{\text {To }}$ y $5^{\text {to }}$ de secundaria se llegó a la conclusión que el $95 \%$ de los encuestados si continuara con sus estudios de nivel universitario y un $6 \%$ de ellos estudiara la carrera profesional de enfermería.

\section{CONCLUSIONES}

En la revisión del estudio, se ha encontrado que en el Perú existe una mala distribución del personal de salud, identificando a algunos departamentos la necesidad de profesionales en enfermería.

La revisión de la literatura nos habla que el profesional de enfermería debe orientar su conocimiento al área de la gerontología, abarcando temas de promoción y prevención de la salud.

La carrera de enfermería es una profesión con futuro, debido a la necesidad de profesionales de la salud.

Existe un alto porcentaje de jóvenes que continuaran sus estudios de nivel universitario, eligiendo el $6 \%$ del total la carrera de enfermería.

\section{REFERENCIAS BIBLIOGRÁFICAS}

1. ASPEFEEN. Asociación Peruana de Facultades y Escuelas de Enfermería (Año sin especificar). "Perfil Educativo de la Egresada de los Programas de Pre Grado en base a Competencias. Disponible en: http://www.aspefeen.org.pe/acredita/reultados/ index.php

2. Cabrera, C. (2013). Facultad de Medicina de la USMP. Disponible en: http://www. usmp.edu.pe/fobstetri/escuelas/enfermeria/index.php

3. Educared Perú. (Año sin especificar). Perspectivas de la educación superior y necesidades del mercado ocupacional. Fundación Telefónica. Disponible en: http:// www.educared.edu.pe/directivos/articulo/183/perspectivas-de-la-educacionsuperior-y-necesidades-del-mercado-ocupacional/

4. Guerrero, D. (2012). Enfermería, una carrera que promueve la salud del adulto mayor. Disponible en: http://www.carrerasconfuturo.com/2012/09/05/enfermeriauna-carrera-que-promueve-la-salud-del-adulto-mayor/ 
5. Guerrero, O. (2012). Gestión con ética en la educación en el Perú. Disponible en: http://www.ipae.pe/media/201211/gestionconeticaenlaeducacion.pdf

6. International Council of Nurses (2013). International Nursing Review. Disponible en: http://www.icn.ch/publications/international-nursing-review-inr/

7. Ministerio de Salud del Perú (2013). Enfermedades que aquejan a la población, datos estadísticos. Disponible en: http://www.minsa.gob.pe/

8. Ministerio de Salud del Perú (2013). Recursos humanos del sector salud, datos estadísticos. Disponible en: http://www.minsa.gob.pe/

9. Piscoya, H. (2013). Formación universitaria vs Mercado laboral. Asamblea Nacional de Rectores. Lima, Perú. 